\title{
The Medial Subthalamic Nucleus Border as a New Anatomical Reference in Stereotactic Neurosurgery for Parkinson's Disease
}

\author{
Erik Bolier ${ }^{a, b} \quad$ Maarten Bot ${ }^{b}$ Pepijn van den Munckhof ${ }^{b} \quad$ Gian Pal $^{a}$ \\ Sepehr Sanic Leo Verhagen Metman ${ }^{a}$
}

${ }^{a}$ Department of Neurological Sciences, Rush University Medical Center, Chicago, IL, USA; ${ }^{b}$ Department of Neurosurgery, Amsterdam University Medical Centers, Academic Medical Center, Amsterdam, The Netherlands;

'Department of Neurosurgery, Rush University Medical Center, Chicago, IL, USA

\section{Keywords}

Deep brain stimulation · Parkinson's disease ·

Subthalamic nucleus · Red nucleus · T2-weighted

imaging · Susceptibility-weighted imaging

\begin{abstract}
Introduction: The intersection of Bejjani's line with the welldelineated medial subthalamic nucleus (STN) border on MRI has recently been proposed as an individualized reference in subthalamic deep brain stimulation (DBS) surgery for Parkinson's disease (PD). We, therefore, aimed to investigate the applicability across centers of the medial STN border as a patient-specific reference point in STN DBS for PD and explore anatomical variability between left and right mesencephalic area within patients. Furthermore, we aim to evaluate a recently defined theoretic stimulation "hotspot" in a different center. Methods: Preoperative 3-Tesla T2 and susceptibility-weighted images (SWI) were used to identify the intersection of Bejjani's line with the medial STN border in left and right mesencephalic area. The average stereotactic coordinates of the center of stimulation relative to the medial STN border were compared with the predefined theoretic stimulation "hotspot." Results: Fifty-four patients provided 108 stereotactic coordinates of medial STN borders on both se-
\end{abstract}

karger@karger.com www.karger.com/sfn

Karger $\frac{1}{\%}$

GOPEN ACCESS
(C) 2020 The Author(s)

Published by S. Karger AG, Basel

This is an Open Access article licensed under the Creative Common Attribution-NonCommercial-4.0 International License (CC BY-NC) (http://www.karger.com/Services/OpenAccessLicense), applicable to the online version of the article only. Usage and distribution for commercial purposes requires written permission. quences. Significant difference in means was found in the $\mathrm{Y}$-(anteroposterior) and Z-(dorsoventral) directions (T2 vs. SWl; $p<0.001$ ). Mean coordinates in the $Y$-(anteroposterior) direction differed significantly between left and right mesencephalic area (T2: $p<0.001$; SWI: $p=0.021)$. Sixty-six DBS leads were placed in 36 patients that had finished stimulation programming, and the average stereotactic coordinates of the center of stimulation relative to the medial STN border on T2 sequences were $3.1 \mathrm{~mm}$ lateral, $0.7 \mathrm{~mm}$ anterior, and $1.8 \mathrm{~mm}$ superior, in proximity of the predefined theoretic stimulation "hotspot." Conclusion: The medial STN border is applicable across centers as a reference point for STN DBS surgery for PD and seems suitable in order to account for interindividual and intraindividual anatomical variability if one is aware of the discrepancies between T2-weighted imaging and SWI.

(c) 2020 The Author(s)

Published by S. Karger AG, Basel

\section{Introduction}

Deep brain stimulation (DBS) of the dorsolateral sensorimotor part of the subthalamic nucleus (STN) is considered the most effective surgical treatment for medically refractory Parkinson's disease (PD) [1]. However, 
clinical outcome after STN DBS varies widely between patients [2-5]. In part, this may be explained by individual anatomical variability of the STN combined with poor visualization of the dorsolateral sensorimotor part of the STN with current imaging techniques $[6,7]$.

The midcommissural point (MCP) is currently the anatomical reference point in stereotactic neurosurgery for STN DBS. By identifying the anterior commissure (AC) and posterior commissure (PC) on MRI, stereotactic coordinates relative to the MCP are used for preoperative planning, intraoperative targeting and DBS lead placement, and postoperative localization of the active DBS lead contacts. The interpretation of stereotactic coordinates without accounting for patient's anatomical variability, however, is of questionable merit [8-12].

In a recent publication, Bot et al. [12] proposed the medial STN border (defined as the intersection of Bejjani's line [13] with the medial border of the STN) as a new, individualized, reference point that is well delineated on standard MR imaging [12]. In their study, the authors found a significant correlation between clinical improvement and the distance to the active stimulation contacts from this medial STN reference point; in contrast, such a correlation was not found between clinical improvement and distance to active stimulation contacts from the MCP. The authors went on to define a theoretic stimulation "hotspot" within the STN, at $2.8 \mathrm{~mm}$ lateral, $1.7 \mathrm{~mm}$ anterior, and $2.5 \mathrm{~mm}$ superior to the medial STN border. More recently, using the same methods in a second, larger patient cohort, this theoretic stimulation "hotspot" was refined and pinpointed to $2.6 \mathrm{~mm}$ lateral, $0.7 \mathrm{~mm}$ anterior, and $1.9 \mathrm{~mm}$ superior to the medial STN border on T2-weighted imaging (throughout this manuscript referred to as "predefined theoretic stimulation 'hotspot"'; Bot et al. Deep brain stimulation for Parkinson's disease: refining the optimal location within the subthalamic nucleus. Unpublished data and abstract presentation at XXIIIrd Congress of the European Society of Stereotactic and Functional Neurosurgery, Edinburgh, UK, September 28, 2018). Again, the medial STN border proved superior compared to the MCP as anatomical reference for correlation of DBS location and motor improvement. However, the applicability of the medial STN border as a reference point in DBS surgery for PD across different centers remains unknown.

As acknowledged by the authors, the mean stereotactic coordinates of the medial STN border $(N=65,3$-Tesla T2-weighted images) varied considerably, at $9.2 \pm 1.1$ (range 6.8-11.5) mm lateral, $2.8 \pm 0.8$ (range 1.5-5.3) $\mathrm{mm}$ posterior, and $4.2 \pm 1.0$ (range $2.0-6.1$ ) $\mathrm{mm}$ inferior to the
MCP [12]. This supports the need to account for interindividual anatomical variability in STN DBS surgery. In addition, morphometric variability of the STN and the red nucleus (RN) between left and right mesencephalic area within patients with advanced PD has been reported [14]. This suggested intraindividual anatomical variability could be relevant in localizing the medial STN border in DBS surgery for PD.

As susceptibility-weighted imaging (SWI) gained interest in DBS surgery for PD due to its better visualization of the STN compared to T2-weighted imaging, discrepancies between T2-weighted imaging and SWI have been a subject of study $[6,7,15,16]$. In localizing the medial STN border, the correspondence between T2-weighted imaging and SWI remains unclear. When employing the medial STN border as an individualized anatomical reference point in DBS surgery for PD, however, this information is of value.

In the current study, we aimed to investigate the applicability across centers of the medial STN border as a new, individual reference point on both 3-Tesla T2weighted imaging and SWI, and explored anatomical variability between left and right mesencephalic area within patients. In addition, we aimed to evaluate the recently refined theoretic stimulation "hotspot" in PD patients that underwent STN DBS surgery in a different center.

\section{Materials and Methods}

Patient Selection and Clinical Data Collection

We selected patients with PD who underwent STN DBS at Rush University Medical Center (RUMC) between October 2016 and August 2018. We reviewed medical charts and preoperative imaging used for the stereotactic procedure. Patients were included in the current analysis if preoperative MR imaging included both T2weighted and susceptibility-weighted (SWI) sequences. Patients with a history of any intracranial surgery were excluded. We extracted clinical data comprising age, gender, disease duration (defined as years between symptom onset and date of surgery), preoperative levodopa responsiveness (change in UPDRS-III scores between $\mathrm{OFF}$ and $\mathrm{ON}$ medication), the levodopa-equivalent daily dose (LEDD) [17] at baseline and follow-up, and active DBS lead contact(s).

\section{Surgical Procedure}

All patients underwent awake, single-track MER-guided, frame-based DBS lead placement under local anesthesia. One to 2 weeks prior to the day of surgery, patients underwent MRI (Magnetom Verio 3-Tesla, Siemens, Munich, Germany). Post-contrast $3 \mathrm{D}$ volumetric T1-weighted imaging, T2-weighted imaging, and SWI were merged, and target planning was performed using the StealthStation S7 equipped with Framelink software (Medtronic, 
Minneapolis, MN, USA). Landmarks were set by identification of the $\mathrm{AC}, \mathrm{PC}$, and midline on the post-contrast $3 \mathrm{D}$ volumetric $\mathrm{T} 1$ weighted images. Target planning of the dorsolateral part of the STN was based on standard stereotactic coordinates relative to the MCP (12 mm lateral; $4 \mathrm{~mm}$ posterior; $4 \mathrm{~mm}$ inferior) with optimization based on visualization of the RN and STN on T2-weighted imaging and SWI. Trajectory planning was based on standard angles $\left(15-20^{\circ}\right.$ from the mid-sagittal plane and $60^{\circ}$ from the axial plane) with optimization using the post-contrast T1 sequence in probes eye view to localize the entry point on a gyrus and avoid penetration of sulci, ventricles, and blood vessels.

On the day of surgery, subsequent to frame placement (Leksell stereotactic arc and frame G; Elekta, Stockholm, Sweden), computed tomography (CT) was performed and co-registered with the preoperative images. Prior to dural opening, intraoperative CTguided extrapolation of the guide tube to the targeted depth was performed (O-arm; Medtronic, Minneapolis, MN, USA) after which, if needed, adjustments on the X-Y stage were made [18, 19]. Single-track MER (Alpha Omega, Nazareth, Israel) through the central channel was performed by a neurologist to confirm or refine targeting by identifying the electrophysiological dorsal and ventral STN border [20], assessing for presence or absence of kinesthetic cells $[8,21-24]$ and performing test stimulation to assess thresholds for therapeutic effect and side effects. In each hemisphere, intraoperative CT (iCT) images were obtained to evaluate the accuracy of the first microelectrode track, as well as final DBS lead placement. Generally, implantable pulse generators were placed 1 week after DBS lead placement.

\section{Identification of the Medial STN Border}

According to methods previously described by Bot et al. [12], we identified the medial STN border by determining the axial plane in which the $\mathrm{RN}$ was visualized at its maximum diameter, using both axial- and coronal-orientated imaging. In this plane, a crosshair was used to draw a line coinciding with the anterior border of the RN, perpendicular to the AC-PC line (Bejjani's line [13]). The point of intersection with the STN was localized and stereotactic coordinates relative to the MCP were retrieved and defined as the medial STN border (Fig. 1). If more than 1 plane depicted the $\mathrm{RN}$ at its maximum diameter, the plane closest to 4 $\mathrm{mm}$ below MCP was chosen. This was done by consensus from 2 reviewers (EB and LVM) for both left and right mesencephalic area on 3-Tesla T2-weighted sequences and SWI. For optimal resolution window to depict STN borders a level of 820 and a width range of 1,080-1,180 was chosen for T2-weighted images. For SWI, a level of 280 and a width of 264 were chosen for all images.

The parameters for T2-weighted images were as follows: repetition time 5,000 ms; echo time $70 \mathrm{~ms}$; slice thickness $2 \mathrm{~mm}$; voxel size $0.7 \times 0.7 \times 2.0 \mathrm{~mm}$; and acquisition time $8: 47 \mathrm{~min}$. For SWI, the parameters were as follows: repetition time $30 \mathrm{~ms}$; echo time $20 \mathrm{~ms}$; slice thickness $1 \mathrm{~mm}$; voxel size $0.5 \times 0.5 \times 1.0 \mathrm{~mm}$; and acquisition time 11:40 $\mathrm{min}$.

\section{Evaluation of the Stimulation Hotspot}

Stereotactic coordinates of the center of the active contact at final stimulation programming were retrieved using the co-registered $\mathrm{iCT}$ used for DBS lead accuracy evaluation and defined as the center of stimulation. When 2 or more contact points were activated simultaneously, for instance in double monopolar, bipolar, or interleaving stimulation, the midpoint between active contacts was set as center

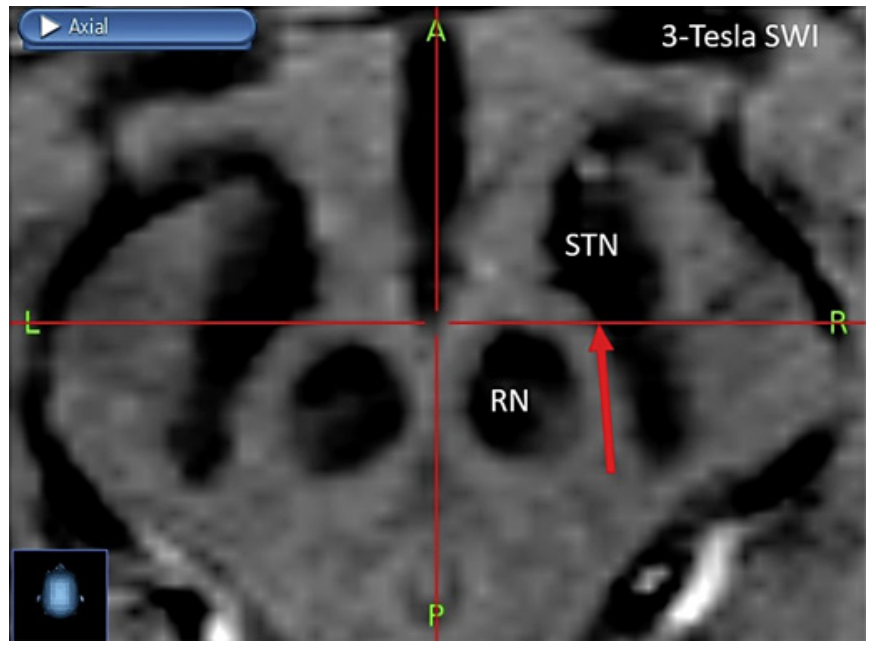

Fig. 1. Defining the medial STN border (red arrow) by drawing a line perpendicular to the AC-PC line at the anterior border of the $\mathrm{RN}$; at its biggest diameter on axial plane on 3-Tesla SWI. STN, subthalamic nucleus; AC, anterior commissure; PC, posterior commissure; RN, red nucleus; SWI, susceptibility-weighted imaging.

of stimulation. The stereotactic coordinates of the center of stimulation relative to the medial STN border were averaged and compared with the predefined theoretic stimulation "hotspot" (2.6 lateral, 0.7 anterior, and 1.9 superior to the medial STN border).

\section{Statistical Analysis}

After testing for normality using the Shapiro-Wilk test (W statistic $>0.900$ ), numeric data were presented as mean with standard deviation. We compared mean coordinates between T2 and SWI sequences using the paired-samples $T$ test. To compare mean coordinates between the current study and the study of Bot et al. [12], and between left and right hemisphere, we used the independentsamples $T$ test, after conversion of left (negative) X-coordinates into absolute values. The average active contact coordinates were compared to the predefined theoretic stimulation "hotspot" using the 1-sample $T$ test. SPSS Statistics 25 (IBM Corp., Armonk, NY, USA) was used for all statistical analyses. A $p$ value of $<0.05$ was considered statistically significant.

\section{Results}

116 STN DBS leads were placed in 65 patients. Fiftyone patients underwent bilateral STN DBS. Fourteen procedures were unilateral, whereof 8 in the left hemisphere and 6 in the right hemisphere. An overview of baseline patient characteristics is presented in Table 1. Fifty-four patients out of 65 (83\%) were included in current analysis, providing 108 stereotactic coordinates of medial STN borders on both T2-weighted imaging and SWI. A flowchart is presented in Figure 2, including reasons for exclusion. 
Table 1. Patient characteristics

\begin{tabular}{lc}
\hline Baseline clinical characteristics $(n=65)$ & \\
Female & $14(22 \%)$ \\
Age, years & $64(59-67)$ \\
Disease duration, years & $9(6-14)$ \\
L-dopa responsiveness, $\%$ & $52 \pm 19$ \\
LEDD, mg & $1,041(650-1,485)$ \\
Intraoperative characteristics $n=65)$ & $51(79 \%)$ \\
Bilateral STN & $8(12 \%)$ \\
Unilateral left STN & $6(9 \%)$ \\
Unilateral right STN & $1.4 \pm 0.6$ \\
Number of MER tracks & $4.8 \pm 2.1$ \\
"First-pass" STN length, mm & $300(100-510)$ \\
Outcome $(n=42)$ & $700(440-1,208)$ \\
LEDD, mg & $76(50-88)$ \\
Absolute LEDD reduction, mg & \\
LEDD reduction, $\%$ &
\end{tabular}

Numeric data presented in median (interquartile range) or mean \pm standard deviation. LEDD, levodopa-equivalent daily dose; STN, subthalamic nucleus.

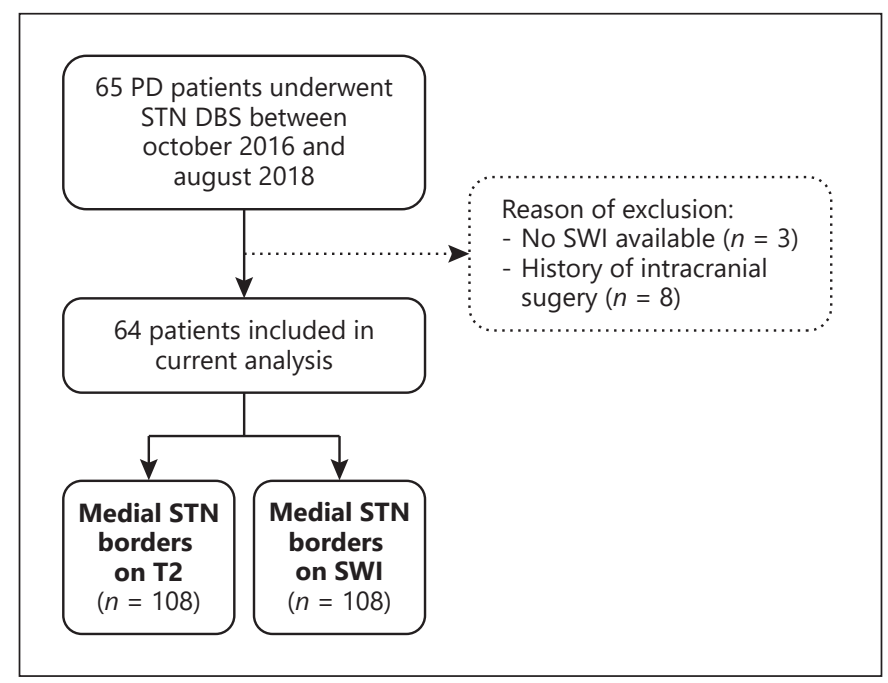

Fig. 2. Flowchart. PD, Parkinson's disease; STN, subthalamic nucleus; DBS, deep brain stimulation; SWI, susceptibility-weighted imaging.

\section{Medial STN Border}

Reproducibility

No statistical difference in mean stereotactic coordinates of the medial STN border on T2-weighted images between the current study and the study of Bot et al. [12] was found in all 3 directions (independent $\mathrm{t}[171]=1.6$, $p=0.12$ in the $\mathrm{X}$-[lateral] direction; $\mathrm{t}[171]=1.12, p=0.26$ in the $\mathrm{Y}$-[anteroposterior] direction, and $\mathrm{t}[171]=1.75$, $p=0.08$ in the $\mathrm{Z}$-[dorsoventral] direction).
Table 2. Medial STN border coordinates on 3-Tesla T2 and SWI sequences

\begin{tabular}{|c|c|c|c|}
\hline \multirow[t]{2}{*}{ Coordinate } & \multicolumn{2}{|c|}{$\begin{array}{l}\text { Average coordinates medial STN } \\
\text { border }(n=108)\end{array}$} & \multirow[t]{2}{*}{$p$ value } \\
\hline & $\mathrm{T} 2$ & SWI & \\
\hline $\mathrm{X}$ & $8.9 \pm 1.0$ & $9.0 \pm 1.1$ & ns \\
\hline Y & $-2.6 \pm 0.7$ & $-2.3 \pm 1.0$ & $<0.001$ \\
\hline $\mathrm{Z}$ & $-4.0 \pm 0.5$ & $-4.5 \pm 0.7$ & $<0.001$ \\
\hline
\end{tabular}

Data presented in mean \pm SD. ns, not significant; STN, subthalamic nucleus; SWI, susceptibility-weighted imaging.

\section{T2 versus SWI Sequences}

Mean stereotactic coordinates of the medial STN border as defined on T2-weighted images and SWI are presented in Table 2. A statistical significant difference in means between T2-defined and SWI-defined coordinates wasfound in theY-(anteroposterior) and Z-(dorsoventral) directions (paired samples $\mathrm{t}[107]=-4.1, p<0.001$ and $\mathrm{t}[107]=7.8, p<0.001$, respectively). The anatomical location of the STN across individuals varied, as coordinates ranged from 6.1 to $10.9 \mathrm{~mm}$ in the $\mathrm{X}$-(mediolateral) direction, from -4.3 to $-1.0 \mathrm{~mm}$ in the $\mathrm{Y}$-(anteroposterior) direction, and from -5.4 to $-2.2 \mathrm{~mm}$ in the Z(dorsoventral) direction on T2-weighted imaging. When defining the medial STN border on SWI, these ranges were $6.2-11.6 \mathrm{~mm}$ in the $\mathrm{X}$-(mediolateral) direction, -4.9 to $-0.1 \mathrm{~mm}$ in the $\mathrm{Y}$-(anteroposterior) direction, and -6.0 to $-2.2 \mathrm{~mm}$ in the $\mathrm{Z}$-(dorsoventral) direction.

\section{Left versus Right Mesencephalic Area}

When defining the medial STN border on T2-weighted imaging, mean stereotactic coordinates in the Y(anteroposterior) direction differed significantly between left and right mesencephalic area (independent $\mathrm{t}[106]=$ 3.6, $p<0.001$; Table 3), reflecting intraindividual anatomical variability. In 44 cases $(81.5 \%)$, the medial STN border was defined more anteriorly on the right side than on the left; in 10 cases $(22.7 \%)$, the difference was $>1.0$ $\mathrm{mm}$. Of note, these findings were due to the anterior border of the RN being located more anteriorly in the right mesencephalic area than in the left.

Consistently, this relative anterior location of the medial STN border in the right mesencephalic area was also found on SWI (independent $t[106]=-2.3, p=0.021$; Table 4$)$. In 10 out of the 39 patients (25.6\%) with a more anterior defined medial STN border on the right mesencephalic area compared to the left, the difference was $>1.0$ 
Table 3. Medial STN border coordinates per brain side on 3-Tesla T2 sequences

\begin{tabular}{llll}
\hline Coordinate & \multicolumn{2}{l}{$\begin{array}{l}\text { Average coordinates medial STN } \\
\text { border on T2 }\end{array}$} & \\
\cline { 2 - 3 } & $\begin{array}{l}\text { left brain side } \\
(n=54)\end{array}$ & $\begin{array}{l}\text { right brain side } \\
(n=54)\end{array}$ & \\
\hline $\mathrm{X}$ & $-9.0 \pm 1.0$ & $8.9 \pm 0.9$ & $\mathrm{~ns}$ \\
$\mathrm{Y}$ & $-2.9 \pm 0.7$ & $-2.4 \pm 0.7$ & $<0.001$ \\
$\mathrm{Z}$ & $-4.0 \pm 0.5$ & $-3.9 \pm 0.5$ & $\mathrm{~ns}$ \\
\hline
\end{tabular}

Data presented in mean \pm SD. ns, not significant; STN, subthalamic nucleus.

Table 5. Stimulation "hotspot" evaluation

\begin{tabular}{llll}
\hline Coordinate & \multicolumn{2}{l}{$\begin{array}{l}\text { Average center of stimulation coordinates } \\
\text { relative to the medial STN border }\end{array}$} & \\
\cline { 2 - 3 } & $\begin{array}{l}\text { current cohort } \\
(n=66)\end{array}$ & $\begin{array}{l}\text { predefined } \\
\text { "hotspot" }\end{array}$ & \\
\hline $\mathrm{X}$ & $3.1 \pm 1.2$ & 2.6 & 0.003 \\
$\mathrm{Y}$ & $0.7 \pm 1.1$ & 0.7 & $\mathrm{~ns}$ \\
$\mathrm{Z}$ & $1.8 \pm 1.4$ & 1.9 & $\mathrm{~ns}$ \\
\hline
\end{tabular}

Data presented in mean \pm SD. ns, not significant; STN, subthalamic nucleus.

mm. Taken T2 and SWI sequences together, 3 cases had a $>1.0 \mathrm{~mm}$ anterior medial STN border on the right side compared to the left on both sequences.

\section{Evaluation of the Stimulation Hotspot}

Thirty-six out of the 54 included patients (66.7\%) had finished stimulation programming. Sixty-six DBS leads were placed in these patients ( 30 bilateral, 6 unilateral), of which a majority of 41 leads (62.1\%) were activated in monopolar configuration. Of the other DBS leads, 9 (13.6\%) were activated in bipolar, 8 (12.1\%) in double monopolar, and $8(12.1 \%)$ in interleaving configurations. The average stereotactic coordinates of the center of stimulation relative to the medial STN border on T2 sequences were $3.1 \pm$ $1.2 \mathrm{~mm}$ lateral, $0.7 \pm 1.1 \mathrm{~mm}$ anterior, and $1.8 \pm 1.4 \mathrm{~mm}$ superior, in proximity of the predefined theoretic stimulation hotspot (Table 5). Thirty-two (48.5\%) of the DBS leads were placed using the central channel of the BenGun. Other DBS leads were placed in the posterior $(n=6)$, medial $(n=5)$, and lateral $(n=3)$ channel using the "+" configuration of the BenGun, and in the posteromedial
Table 4. Medial STN border coordinates per brain side on 3 Tesla SWI

\begin{tabular}{llll}
\hline Coordinate & \multicolumn{2}{l}{$\begin{array}{l}\text { Average coordinates medial STN } \\
\text { border on SWI }\end{array}$} & \\
\cline { 2 - 3 } & $\begin{array}{l}\text { left brain side } \\
(n=54)\end{array}$ & $\begin{array}{l}\text { right brain side } \\
(n=54)\end{array}$ & \\
\hline $\mathrm{X}$ & $-9.0 \pm 1.1$ & $9.0 \pm 1.0$ & $\mathrm{~ns}$ \\
$\mathrm{Y}$ & $-2.6 \pm 1.0$ & $-2.1 \pm 0.9$ & 0.021 \\
$\mathrm{Z}$ & $-4.5 \pm 0.7$ & $-4.4 \pm 0.7$ & $\mathrm{~ns}$ \\
\hline
\end{tabular}

Data presented in mean \pm SD. ns, not significant; STN, subthalamic nucleus; SWI, susceptibility-weighted imaging.

Table 6. BenGun channel used for DBS lead placement

\begin{tabular}{lc}
\hline BenGun channel used for DBS lead $(n=66)$ \\
\hline Central & $32(48.5 \%)$ \\
Anterior & $0(0 \%)$ \\
Lateral & $3(4.5 \%)$ \\
Posterior & $6(9.1 \%)$ \\
Medial & $5(7.6 \%)$ \\
Anterolateral & $0(0 \%)$ \\
Anteromedial & $3(4.5 \%)$ \\
Posterolateral & $4(6.1 \%)$ \\
Posteromedial & $4(6.1 \%)$ \\
Stage move & $7(10.6 \%)$ \\
Missing data & $2(3 \%)$ \\
\hline
\end{tabular}

DBS, deep brain stimulation.

$(n=4)$, posterolateral $(n=4)$, and anteromedial $(n=3)$ channel using the " $\mathrm{x}$ " configuration of the BenGun [25]. In 7 instances, adjustment of the head stage (stage move) was performed prior to DBS lead placement, since adjustment could not be achieved by using 1 of the predefined channels of the BenGun (see Table 6).

\section{Discussion}

In the current study, we evaluated the applicability of a new, individual anatomical reference point in STN DBS for PD: the medial STN border. Using recently described methods [12], we were able to reproduce similar stereotactic coordinates of the medial STN border in patients with PD that underwent surgery in a different center. Furthermore, the active contacts of the patients in the current study were located in close proximity of the predefined 
theoretic stimulation "hotspot." Therefore, our results suggest that the medial STN border as an anatomical reference point is suitable in order to account for individual anatomical variability in STN DBS, if one is aware of the discrepancies between T2-weighted imaging and SWI. Interestingly, our results imply that within PD patients, an anatomical difference between left and right position of the $\mathrm{RN}$ exists.

\section{The Medial STN Border}

In concordance with previous results [12], individual stereotactic coordinates of the defined medial STN borders varied considerably in all 3 directions, underlining the need to account for anatomical variability between patients. Above that, in the current study we found a statistical difference in mean Y-(anteroposterior) coordinates between left and right medial STN border, suggesting that accounting for anatomical variability within patients is also necessitated.

Garcia-Garcia et al. [11] created an adaptable 3D atlas in order to account for individual anatomical variability in determining the optimal stimulation location. However, this technique is based on the Morel atlas which does not provide bilateral anatomical information and required various software packages that may not be available for all DBS centers. Using the medial STN border as an anatomical reference point, which is easily defined by the coinciding point of the well-known Bejjani line with the delineation of the medial STN border visualized on conventional imaging, can overcome both these issues.

\section{T2 versus SWI}

Although SWI has gained interest in DBS surgery for PD for its better visualization of the STN compared to T2weighted imaging, the reliability of SWI has been a point of discussion $[6,7,15,16]$. For instance, discrepancies are found in defining dorsal and ventral STN borders between SWI and MER [7], especially when using lower field strengths [6]. In our current study we found discrepancies between 3-Tesla T2 and SWI sequences regarding medial STN border coordinates, that reached statistical significance in the $\mathrm{Y}$-(anteroposterior) and Z(dorsoventral) directions. Whether the use of ultrahigh field strength (7-Tesla) MRI improves the discrepancies between T2-weighted imaging and SWI remains uncertain. Therefore, when implementing the medial STN border as a new anatomical reference in STN DBS, 1 should be aware of discrepancies in visualization of the basal ganglia between T2-weighted imaging and SWI, for instance in defining an optimal stimulation location or localizing kinesthetic cell clusters. Since the electrophysiological medial STN border is generally not sought using MER in DBS surgery for PD, differences in reliability between T2weighted imaging and SWI could not be measured in the current study.

\section{The Stimulation Hotspot}

Although a statistical difference in means was found in the X-(lateral) direction between the theoretic stimulation "hotspot" defined by Bot et al. [12] and the stimulation location of the patients in the current study, we consider this negligible for 2 reasons. First, the coordinates represent a center within an electric potential field rather than a pinpoint "hotspot." The volume of tissue activated likely exceeds $0.5 \mathrm{~mm}$ and, above that, the diameter of a contact is more than $1 \mathrm{~mm}$. Second, both stimulation locations were found in the dorsolateral part of the STN. Taken together, this suggests that the stimulation "hotspot" (located $2.6 \mathrm{~mm}$ lateral, $0.7 \mathrm{~mm}$ anterior, and $1.9 \mathrm{~mm}$ superior to the medial STN border) is applicable in PD patients that underwent STN DBS surgery across centers.

Alternatively, the stimulation "hotspot" could be evaluated by correlating the 3-dimensional distance between the hotspot coordinates and the center of stimulation of the patients with an UPDRS-III reduction at follow-up. At the senior authors' institution, however, the UPDRSIII is not standardly measured at follow-up, given the vast majority of the patients have to travel a considerable amount of time and assessment of the UPDRS-III is time consuming. Therefore, the LEDD reduction at follow-up is used to evaluate the effectiveness of stimulation. However, the LEDD reduction is not a lateralized outcome, whereas the majority of PD patients that undergo DBS surgery receive bilateral treatment. In case of bilateral stimulation, it is not possible to determine to what extent stimulation in each hemisphere contributes to reduction in LEDD. We, therefore, considered the LEDD reduction at follow-up not suitable as a surrogate outcome measure. The reduction in lateralized UPDRS-III score seems suitable as an outcome measure, although stimulation in 1 hemisphere can have clinical effect in the ipsilateral body side as well $[26,27]$.

Andrade-Souza et al. [28] concluded that targeting based on the borders of the $\mathrm{RN}$ is more accurate compared to the established indirect targeting (AC-PC based) and modified direct targeting $(3 \mathrm{~mm}$ above the center of the STN on coronal sections using T2-weighted images). As the authors point out, the distance between their RN- 
based target and the location of the optimal contact was nonetheless more than $3 \mathrm{~mm}$ on average and therefore electrophysiological determination of the final electrode position was recommended. Our patient-specific reference point (also RN based) allows to further optimize targeting within the dorsolateral STN. We, therefore, encourage other centers to localize a stimulation "hotspot" by calculating the stereotactic distance between active DBS lead contacts and the medial STN border using optimal responders within their patient population, and compare the results to the predefined theoretic stimulation "hotspot" by Bot et al. [12] (2.6 mm lateral, $0.7 \mathrm{~mm}$ anterior, and $1.9 \mathrm{~mm}$ superior to the medial STN border on T2-weighted imaging). Eventually, this could enable direct medial STN border-based targeting using data from optimal responders worldwide and increase overall clinical effectiveness of STN-DBS in future patients with Parkinson's disease.

\section{The Red Nucleus}

Since we defined the medial STN border using the anterior border of the $\mathrm{RN}$ at its maximal diameter, our findings of a statistically different average left and right Ycoordinate, on both T2-weighted imaging and SWI, directly reflect a more anterior position of the anterior border of the $\mathrm{RN}$ in the right mesencephalic area compared to the left. In roughly one out of 4 patients, the right $\mathrm{RN}$ was more than $1 \mathrm{~mm}$ anterior than the left, which we consider clinically relevant in stereotactic DBS surgery.

These findings raise the question to what extent the anatomical position of the $\mathrm{RN}$ (mesencephalic origin) and the STN (diencephalic origin) relate. Also, it remains uncertain whether these findings are disease and/or age related. All the patients included in current study were right handed (data not provided).

One study of Xiao et al. [14] investigated morphometric variability of the STN and RN in patients with advanced $\mathrm{PD}$. In concordance with our findings, the authors report a more anteriorly mean distance between the geometric center of the right $\mathrm{RN}$ to the MCP than the left RN. Similar differences were found regarding the STN, also when using the center of mass to calculate the distance to the MCP; however, left and right measurements were not directly statistically compared to each other but independently to the Talairach [29] atlas.

Various reports provide data on left and right volumetric properties of basal ganglia, including the STN and RN [10, 14, 30-32]. However, analysis of volumetric variability between left and right hemispheres was either not significant $[10,30,31]$ or did not lay within the scope of the study [32]. In theory, however, a volumetric change could occur independent from positional or morphometric changes.

\section{Study Limitations}

Although the use of intraoperative CT to determine lead placement accuracy in deep brain stimulation surgery has been validated [33-35], inherent measurement error has been reported [34]. Therefore, fusion of preoperative MRI and iCT images were carefully inspected for each case to reduce merging error. In addition, standard surgical precautions were taken to limit the effect of brain shift on lead placement accuracy [36, 37]. Finally, it should be noted that the difference in slice thickness values of our study potentially limits our methodology. It has previously been suggested, however, that these values would provide a comparable degree of accuracy [7, 38].

\section{Conclusion}

The medial STN border is applicable across centers as a new anatomical reference point for STN DBS surgery for $\mathrm{PD}$, and seems suitable in order to account for interindividual and intraindividual anatomical variability if one is aware of the discrepancies between T2-weighted imaging and SWI. The clinical implications of our findings of a more anteriorly positioned $\mathrm{RN}$ in the right mesencephalic area compared to the left should be further investigated.

\section{Statement of Ethics}

The study was approved by the Rush University Institutional Review Board (ID: 18050108; date of approval: June 11, 2018), and informed consent was obtained.

\section{Conflict of Interest Statement}

L.V.M. has received fees for consulting activities, advisory boards, and educational activities from Abbott, AbbVie Inc., Biogen Inc., Boston Scientific, Medtronic Inc., and WorldMed Inc. S.S. has received fellowship training grant support from Medtronic Inc., Abbott, and Boston Scientific. The DBS team of the Academic Medical Center (M.B. and P.V.D.M.) received unrestricted research grants from Medtronic and financial compensation for teaching courses for the European Continued Medical Training Program. 


\section{Funding Sources}

E.B. received financial support from the Vreedefonds Foundation; Parkinson's Foundation, The Netherlands; and Amsterdam Foundation for Promoting Neurosurgical Development.

\section{Author Contributions}

E.B.: conceptualization, methodology, investigation, data curation, formal analysis, visualization, and writing - original draft. M.B.: conceptualization, methodology, and writing - review and editing. P.V.D.M.: project administration, conceptualization, methodology, and writing - review and editing. G.P.: conceptualization, data curation, and writing - review and editing. S.S.: software, resources, data curation, and writing - review and editing. L.V.M.: project administration, conceptualization, methodology, data curation, supervision, and writing - review and editing.

\section{References}

1 Herzog J, Fietzek U, Hamel W, Morsnowski A, Steigerwald F, Schrader B, et al. Most effective stimulation site in subthalamic deep brain stimulation for Parkinson's disease. Mov Disord. 2004;19(9):1050-4.

2 Krack P, Batir A, Van Blercom N, Chabardes S, Fraix V, Ardouin C, et al. Five-year followup of bilateral stimulation of the subthalamic nucleus in advanced Parkinson's disease. N Engl J Med. 2003;349(20):1925-34.

3 Kleiner-Fisman G, Herzog J, Fisman DN, Tamma F, Lyons KE, Pahwa R, et al. Subthalamic nucleus deep brain stimulation: summary and meta-analysis of outcomes. Mov Disord. 2006;21(S14):S290-304.

4 Odekerken VJ, van Laar T, Staal MJ, Mosch A, Hoffmann CF, Nijssen PC, et al. Subthalamic nucleus versus globus pallidus bilateral deep brain stimulation for advanced Parkinson's disease (NSTAPS study): a randomised controlled trial. Lancet Neurol. 2013;12(1): 37-44.

5 Bang Henriksen M, Johnsen EL, Sunde N, Vase A, Gjelstrup MC, Østergaard K. Surviving 10 years with deep brain stimulation for Parkinson's disease: a follow-up of 79 patients. Eur J Neurol. 2016;23(1):53-61.

6 Bot M, Bour L, de Bie RM, Contarino MF, Schuurman PR, van den Munckhof P. Can we rely on susceptibility-weighted imaging for subthalamic nucleus identification in deep brain stimulation surgery? Neurosurgery. 2015;78(3):353-60.

7 Bus S, van den Munckhof P, Bot M, Pal G, Ouyang B, Sani S, et al. Borders of STN determined by MRI versus the electrophysiological STN. A comparison using intraoperative CT. Acta Neurochir. 2018 Feb;160(2):373-83.

8 Romanelli P, Heit G, Hill BC, Kraus A, Hastie T, Brontë-Stewart HM. Microelectrode recording revealing a somatotopic body map in the subthalamic nucleus in humans with Parkinson disease. J Neurosurg. 2004;100(4): 611-8.

9 Caire F, Ranoux D, Guehl D, Burbaud P, Cuny E. A systematic review of studies on anatomical position of electrode contacts used for chronic subthalamic stimulation in Parkinson's disease. Acta Neurochir. 2013; 155(9):1647-54.
10 Keuken MC, Bazin PL, Schäfer A, Neumann J, Turner R, Forstmann BU. Ultra-high 7T MRI of structural age-related changes of the subthalamic nucleus. J Neurosci. 2013;33(11): 4896-900.

11 Garcia-Garcia D, Guridi J, Toledo JB, Alegre M, Obeso JA, Rodríguez-Oroz MC. Stimulation sites in the subthalamic nucleus and clinical improvement in Parkinson's disease: a new approach for active contact localization. J Neurosurg. 2016;125(5):1068-79.

12 Bot M, Schuurman PR, Odekerken VJJ, Verhagen R, Contarino FM, De Bie RMA, et al. Deep brain stimulation for Parkinson's disease: defining the optimal location within the subthalamic nucleus. J Neurol Neurosurg Psychiatry. 2018 May;89(5):493-8.

13 Bejjani BP, Dormont D, Pidoux B, Yelnik J, Damier P, Arnulf I, et al. Bilateral subthalamic stimulation for Parkinson's disease by using three-dimensional stereotactic magnetic resonance imaging and electrophysiological guidance. J Neurosurg. 2000;92(4):615-25.

14 Xiao Y, Jannin P, D’Albis T, Guizard N, Haegelen $\mathrm{C}$, Lalys $\mathrm{F}$, et al. Investigation of morphometric variability of subthalamic nucleus, red nucleus, and substantia nigra in advanced Parkinson's disease patients using automatic segmentation and PCA-based analysis. Hum Brain Mapp. 2014 Sep;35(9):4330-44.

15 McEvoy J, Ughratdar I, Schwarz S, Basu S. Electrophysiological validation of STN-SNr boundary depicted by susceptibility-weighted MRI. Acta Neurochir. 2015;157(12):2129-34.

16 Polanski WH, Martin KD, Engellandt K, von Kummer R, Klingelhoefer L, Fauser M, et al. Accuracy of subthalamic nucleus targeting by T2, FLAIR and SWI-3-Tesla MRI confirmed by microelectrode recordings. Acta Neurochir. 2015;157(3):479-86.

17 Tomlinson CL, Stowe R, Patel S, Rick C, Gray $\mathrm{R}$, Clarke CE. Systematic review of levodopa dose equivalency reporting in Parkinson's disease. Mov Disord. 2010;25(15):2649-53.

18 Kochanski RB, Kerolus MG, Pal G, Metman $\mathrm{LV}$, Sani S. Use of intraoperative CT to predict the accuracy of microelectrode recording during deep brain stimulation surgery. A proof of concept study. Clin Neurol Neurosurg. 2016 Nov;150:164-8.
19 Kochanski RB, Bus S, Pal G, Metman LV, Sani S. Optimization of microelectrode recording in deep brain stimulation surgery using intraoperative computed tomography. World Neurosurg. 2017 Jul;103:168-73.

20 Sterio D, Zonenshayn M, Mogilner AY, Rezai AR, Kiprovski K, Kelly PJ, et al. Neurophysiological refinement of subthalamic nucleus targeting. Neurosurgery. 2002;50(1):58-9.

21 Rodriguez-Oroz MC, Rodriguez M, Guridi J, Mewes K, Chockkman V, Vitek J, et al. The subthalamic nucleus in Parkinson's disease: somatotopic organization and physiological characteristics. Brain. 2001;124(Pt 9):177790.

22 Abosch A, Hutchison WD, Saint-Cyr JA, Dostrovsky JO, Lozano AM. Movement-related neurons of the subthalamic nucleus in patients with Parkinson disease. J Neurosurg. 2002;97(5):1167-72.

23 Starr PA, Theodosopoulos PV, Turner R. Surgery of the subthalamic nucleus: use of movement-related neuronal activity for surgical navigation. Neurosurgery. 2003;53(5):11469.

24 Theodosopoulos PV, Marks WJ, Christine C, Starr PA. Locations of movement-related cells in the human subthalamic nucleus in Parkinson's disease. Mov Disord. 2003;18(7):791-8.

25 Bus S, Pal G, Ouyang B, van den Munckhof P, Bot M, Sani S, et al. Accuracy of microelectrode trajectory adjustments during DBS assessed by intraoperative CT. Stereotact Funct Neurosurg. 2018;96(4):231-8.

26 Germano IM, Gracies JM, Weisz DJ, Tse W, Koller WC, Olanow CW. Unilateral stimulation of the subthalamic nucleus in Parkinson disease: a double-blind 12-month evaluation study. J Neurosurg. 2004;101(1):36-42.

27 Tabbal SD, Ushe M, Mink JW, Revilla FJ, Wernle AR, Hong M, et al. Unilateral subthalamic nucleus stimulation has a measurable ipsilateral effect on rigidity and bradykinesia in Parkinson disease. Exp Neurol. 2008; 211(1):234-42.

28 Andrade-Souza YM, Schwalb JM, Hamani C, Eltahawy H, Hoque T, Saint-Cyr J, et al. Comparison of three methods of targeting the subthalamic nucleus for chronic stimulation in Parkinson's disease. Neurosurgery. 2005;56(2 Suppl l):360. 
29 Talairach J, Tournoux P. Co-planar stereotaxic atlas of the human brain: 3-dimensional proportional system: an approach to cerebral imaging; 1988.

30 Colpan ME, Slavin KV. Subthalamic and red nucleus volumes in patients with Parkinson's disease: do they change with disease progression? Parkinsonism Relat Disord. 2010 Jul; 16(6):398-403.

31 Keuken MC, Bazin PL, Crown L, Hootsmans J, Laufer A, Müller-Axt C, et al. Quantifying inter-individual anatomical variability in the subcortex using $7 \mathrm{~T}$ structural MRI. NeuroImage. 2014;94:40-6.
32 Keuken MC, Bazin PL, Backhouse K, Beekhuizen S, Himmer L, Kandola A, et al. Effects of aging on $\mathrm{T}(1), \mathrm{T}(2)^{*}$, and QSM MRI values in the subcortex. Brain Struct Funct. 2017 Aug; 222(6):2487-505.

33 Shahlaie K, Larson PS, Starr PA. Intraoperative computed tomography for deep brain stimulation surgery: technique and accuracy assessment. Neurosurgery. 2011;68(1 Suppl Operative):114-24.

34 Holloway K, Docef A. A quantitative assessment of the accuracy and reliability of $\mathrm{O}$-arm images for deep brain stimulation surgery. Neurosurgery. 2012;72(1 Suppl Operative): 47-57.

35 Mirzadeh Z, Chapple K, Lambert M, Dhall R, Ponce FA. Validation of CT-MRI fusion for intraoperative assessment of stereotactic accuracy in DBS surgery. Mov Disord. 2014; 29(14):1788-95.
36 van den Munckhof $\mathrm{P}$, Contarino MF, Bour LJ, Speelman JD, de Bie RM, Schuurman PR. Postoperative curving and upward displacement of deep brain stimulation electrodes caused by brain shift. Neurosurgery. 2010; 67(1):49-4

37 Azmi H, Machado A, Deogaonkar M, Rezai A. Intracranial air correlates with preoperative cerebral atrophy and stereotactic error during bilateral STN DBS surgery for Parkinson's disease. Stereotact Funct Neurosurg. 2011;89(4):246-52.

38 Savio SJ, Harrison LC, Luukkaala T, Heinonen T, Dastidar P, Soimakallio S, et al. Effect of slice thickness on brain magnetic resonance image texture analysis. Biomed Eng Online. 2010;9(1):60. 